\title{
ASSOCIAÇÃO ENTRE BRUXISMO DO SONO E PERSONALIDADE DA CRIANÇA SOB A PERCEPÇÃO DOS PAIS/CUIDADORES
}

\section{ASSOCIATION BETWEEN SLEEPING BRUXISM AND CHILD PERSONALITY UNDER THE PERCEPTION OF PARENTS / CAREGIVERS}

\section{ASOCIACIÓN ENTRE BRUXISMO DURANTE Y PERSONALIDAD INFANTIL BAJO LA PERCEPCIÓN DE PADRES / CUIDADORES}

\author{
Carlos Felipe Bonacina ${ }^{1}$, Florense Gabriela da Silva², Camile Aben-athar Lobato da Silva ${ }^{3}$, \\ Camila Vendramini Galatti Abdala ${ }^{4}$, Adriana de Oliveira Lira ${ }^{5}$
}

Submetido: $20 / 04 / 2020$

Aprovado: 26/06/2020

\section{RESUMO}

Objetivo: Avaliar a associação do bruxismo do sono e personalidade das crianças em relação à percepção dos cuidadores. Metodologia: foram incluídos no estudo 93 crianças de 3 a 10 anos, de ambos os sexos e seus respectivos cuidadores que frequentavam uma Clínica escola Universitária. Inicialmente, os cuidadores responderam sobre estado de saúde geral, presença de bruxismo do sono, constatado através do relato dos pais/cuidadores e traços de personalidade da criança. $O$ estudo propôs identificar a percepção dos pais em relação a personalidade dos filhos expressa de forma rotineira: calma, agitada, hiperativa, ansiosa e agressiva. Para a análise do desfecho e variáveis associadas foi utilizado o Teste qui-quadrado considerando $p<0,05-95 \%$ IC. Resultados: observou-se um menor número de relatos da presença do bruxismo do sono em crianças com a personalidade considerada calma $\left(P=0,014^{*}\right)$ e ansiosa $\left(P=0,0016^{*}\right)$. Conclusão: Tanto crianças consideradas calmas quanto as ansiosas apresentaram menos relato de bruxismo do sono, demonstrando não haver nessa amostra associação entre padrão de personalidade e bruxismo do sono.

PALAVRAS CHAVES: Bruxismo do sono; Distúrbios do sono; Criança

\section{ABSTRACT}

Objective: To evaluate the association between sleep bruxism and children's personality in relation to the perception of caregivers. Methodology: 93 children from 3 to 10 years old, of both sexes and their respective caregivers who attended a University School Clinic, were included in the study. Initially, the caregivers answered about general health status, presence of sleep bruxism, verified through the parents/caregivers reports and the child's personality traits. The study proposed to identify the parents' perception in relation to the children's personality expressed in a routine way: calm, agitated, hyperactive, anxious and aggressive. For the analysis of the outcome and associated variables, the chi-square test was used considering $p<0.05-95 \% \mathrm{Cl}$. Results: there was a smaller number of reports of the presence of sleep bruxism in children with a personality considered calm $\left(P=0.014{ }^{*}\right)$ and anxious $\left(P=0.0016{ }^{*}\right)$. Conclusion: Both children considered calm and anxious had less reports of sleep bruxism, demonstrating that there was no association in this sample between personality pattern and sleep bruxism.

KEYWORDS: Sleep bruxism; Sleep disorders; Child

\footnotetext{
1 Professor do Departamento de Odontologia da Universidade UNG - Doutorando do Programa de Pós-graduação em Odontologia Stricto-sensu da Universidade Cruzeiro do Sul - Unicsul

2 Doutoranda do Programa de Pós-graduação em Odontologia Stricto-sensu da Universidade Cruzeiro do Sul - Unicsul

3 Mestranda do Programa de Pós-graduação em Odontologia Stricto-sensu da Universidade Cruzeiro do Sul - Unicsul

4 Professora do Departamento de Odontopediatria da Universidade UNG -

5 Professora do Programa de Pós-graduação em Odontologia Stricto-sensu da Universidade Cruzeiro do Sul - Unicsul
} 


\section{RESUMEN}

Objetivo: evaluar la asociación entre el bruxismo del sueño y la personalidad de los niños en relación con la percepción de los cuidadores. Metodología: 93 niños de 3 a 10 años de edad, de ambos sexos y sus respectivos cuidadores que asistieron a una Clínica de la Escuela Universitaria, fueron incluidos en el estudio. Inicialmente, los cuidadores respondieron sobre el estado de salud general, la presencia de bruxismo del sueño, verificado a través del informe de los padres/cuidadores y los rasgos de personalidad del niño. El estudio propuso identificar la percepción de los padres en relación con la personalidad de los niños expresada de manera rutinaria: tranquila, agitada, hiperactiva, ansiosa y agresiva. Para el análisis del resultado y las variables asociadas, se utilizó la prueba de chi-cuadrado considerando un $p<0.05-95 \%$ IC. Resultados: hubo un número menor de informes de la presencia de bruxismo del sueño en niños con una personalidad considerada tranquila $(P=0.014 *)$ y ansiosa $(P=0.0016 *)$. Conclusión: Ambos niños considerados tranquilos y ansiosos tuvieron menos informes de bruxismo del sueño, lo que demuestra que no hubo asociación en esta muestra entre el patrón de personalidad y el bruxismo del sueño.

PALABRAS CLAVES: Bruxismo del sueño; Transtornos del sueño; niño 


\section{INTRODUÇÃO:}

Por muitos anos o bruxismo foi um termo genérico que abrangia qualquer atividade muscular rítmica e repetitiva da mandíbula que era caracterizado por apertar ou ranger os dentes, podendo ocorrer durante o sono e/ ou vigília. Porém, no ano de 2018 foi realizado um consenso dos pesquisadores mais importantes da área que além da necessária distinção entre os períodos circadianos, também categoriza o tipo da atividade muscular. Sendo assim, o Bruxismo do sono (BS) foi definido como uma atividade dos músculos da mastigação durante o sono, caracterizado por ser rítmica (fásica) ou não rítmica (tônica) e em "indivíduos saudáveis" não é classificado como "distúrbio do movimento ou do sono"1. Os episódios de bruxismo têm origem no sistema nervoso central e a dopamina é o neurotransmissor mais envolvido em tal condição ${ }^{2,3}$.

Atualmente, as evidências cientificas nos mostram que as crianças são mais acometidas pelo quadro do bruxismo do sono, entretanto, a variabilidade entre os números epidemiológicos é ampla, oscilando entre 3,5 a 40,6\% ${ }^{4}$ A imprecisão do diagnóstico, dessa condição parece ser o responsável pela ampla variação nos números, sendo constatado ausência de associação entre o relato dos pais sobre a presença de BS nas crianças com o diagnóstico positivo dessas condições em exame de polissonografia ${ }^{5}$.

O relato dos pais é fundamental e ainda é o instrumento inicial para identificação do Bruxismo do Sono, tanto para finalidade de pesquisa como para a prática clínica, sendo a condição exigida para os três níveis de diagnóstico: possível, onde se obtém apenas o relato do cuidador, provável onde temos além do relato uma avaliação das facetas de desgaste no exame clínico e o definitivo que é a queixa dos pais e o exame de polissonografia, considerado padrão ouro1. Além disso, a informação sobre a frequência de ocorrência do BS melhora a acurácia do diagnóstico, refletindo o grau de acometimento do indivíduo ${ }^{6}$.

Um fator complicador para o diagnóstico possível do bruxismo é que a percepção dos cuidadores em relação ao BS pode estar sujeita a vieses e assim como de outros distúrbios do sono podem passar despercebidos visto as condições ambientais em que a família vive ${ }^{7}$.

A dificuldade no diagnóstico faz com que profissionais ainda tenham abordagens obsoletas em tratar essa condição levando em conta os aspectos psicológicos das crianças principalmente o estresse e ansiedade e acabam esquecendo de avaliar possíveis fatores associados. Diante desse cenário, torna-se importante avaliar se essas condições têm relação com a presença de bruxismo do sono.

\section{MÉTODO}

Foi realizado um estudo transversal, com uma amostra de 93 crianças e seus respectivos cuidadores, de ambos os sexos na faixa etária de 3 a 10 anos, selecionados na Clínica Odontológica da Universidade Cruzeiro do Sul, na cidade de São Paulo, Brasil. O estudo obteve aprovação do Comitê de Ética em Pesquisa da mesma Instituição, (protocolo número: 2. 056.660). Foram excluídas da amostra aquelas crianças que não possuíam dentes em contato com antagonista. A amostra foi alocada em dois grupos, sendo o Grupo Estudo (GE) com bruxismo do sono e o Grupo Controle (GC) sem bruxismo do sono.

Foi realizada entrevista e anamnese com as crianças e seus cuidadores, além da avaliação clínica de presença ou ausência de desgaste dentário. Na avaliação clínica, foi avaliada a borda incisal de dentes anteriores e a face oclusal de dentes posteriores, todos os dentes presentes foram avaliados. Essa avaliação foi realizada na cadeira odontológica, utilizando espelho clinico e luz do refletor, feita por um único examinador calibrado e especialista em Odontopediatria. Os cuidadores responderam perguntas contidas em anamnese, incluindo dor, fadiga ou desconforto nos músculos da mastigação e travamento mandibular ao acordar. Esses aspectos clínicos podem ser relatados pela criança e/ou pelos cuidadores. Além disso, foram incluídas informações sobre aspectos psicológicos da criança. Foi entregue aos pais uma tabela (diário do BS) e a cada noite onde foi percebido o BS, foi marcado com um X no dia correspondente. Foi orientado que o diário fosse preenchido durante 3 semanas e o cuidador devia manter sua rotina normal, dormindo no mesmo ambiente e mantendo os mesmos hábitos que antecederam a pesquisa.

A análise de regressão logística foi realizada para avaliar a associação entre relato de presença ou ausência de BS e aspectos psicológicos da criança. O nível de significância para todas as variáveis foi de $5 \%$. As análises foram realizadas no sofware Stata 13.0. 


\section{RESULTADOS}

A caracterização relacionada a amostra, foi composta de 45 crianças no GE, sendo que 23 crianças $(51,11 \%)$ eram do sexo masculino e 22 crianças eram do sexo feminino (45,83\%). No grupo controle (GC) esses números foram no total 48 crianças, sendo que 22 eram do sexo masculino (48.89\%) e 26 eram do sexo feminino $(54.17 \%)$. Para o sexo do cuidador, foi determinado que esse seria quem levasse a criança nas consultas e tanto no GE quanto no GC esse valor foi dominado pelo sexo feminino. Nos dois grupos, o $\mathrm{N}$ de cuidador feminino foi de 82, enquanto apenas 11 eram do sexo masculino.

Em relação ao estado civil dos cuidadores, no GE $11(37.93 \%)$ eram solteiros e $32(58.18 \%)$ eram casados e 5 cuidadores eram divorciados ou viúvo. No GC 18 $(62.07 \%)$ eram solteiros e $23(41,82 \%)$ eram casados. Apenas 4 eram viúvos ou separados.

Não foram observadas significância estatísticas na variação de dados sociodemográficos, relacionados as famílias das crianças com e sem BS (tabela 1).
Tabela 1: Análise descritiva das variáveis sociodemográficas das crianças e cuidadores

\begin{tabular}{ccccc}
\hline \multirow{2}{*}{$\begin{array}{c}\text { Variáveis } \\
\text { Sociodemográfico }\end{array}$} & $\begin{array}{c}\text { Ausência } \\
\boldsymbol{n}(\%)\end{array}$ & $\begin{array}{c}\text { Presença } \\
\boldsymbol{n}(\%)\end{array}$ & $\begin{array}{c}\text { Total } \\
\boldsymbol{n}\end{array}$ & $\boldsymbol{p}$-valor \\
\hline \multicolumn{5}{c}{ Sexo crianças } \\
\hline Masculino (ref) & $22(48,89)$ & $23(51,11)$ & 45 & 0,611 \\
Feminino & $26(54,17)$ & $22(45,83)$ & 48 & \\
\hline \multicolumn{5}{c}{ Sexo cuidador } \\
Masculino (ref) & $6(54,55)$ & $5(45,45)$ & 11 & 0,664 \\
Feminino & $39(47,56)$ & $43(52,54)$ & 82 & \\
\hline Estado civil cuidador & & & \\
\hline Solteiro (ref) & $11(37.93)$ & $18(62,07)$ & 29 & \\
Casado & $32(58,18)$ & $23(41,82)$ & 55 & \\
Separado & $3(60)$ & $2(40)$ & 5 & 0.221 \\
Viúvo & $2(50)$ & $2(50)$ & 4 &
\end{tabular}


Uma menor prevalência de BS foi observada em crianças com personalidade calma ou ansiosa. Por outro lado, crianças agitadas, hiperativas ou agressivas não foram associadas com a prevalência de bruxismo do sono (tabela 2).

Tabela 2: Análise descritiva das variáveis independentes relacionados à personalidade da criança por presença ou ausência de bruxismo e regressão logística univariada e ajustada entre o desfecho (presença de bruxismo).

\begin{tabular}{|c|c|c|c|c|c|c|c|}
\hline \multirow[b]{2}{*}{ Variáveis } & \multicolumn{3}{|c|}{ Bruxismo } & \multicolumn{2}{|c|}{ OR Univariado } & \multicolumn{2}{|c|}{ OR Ajustado } \\
\hline & $\begin{array}{c}\text { Ausência } \\
n(\%)\end{array}$ & $\begin{array}{c}\text { Presença } \\
n(\%)\end{array}$ & $\begin{array}{c}\text { Total } \\
n\end{array}$ & OR (IC 95\%) & $p$-valor & OR (IC 95\%) & $p$-valor \\
\hline \multicolumn{8}{|c|}{ Personalidade } \\
\hline \multicolumn{8}{|l|}{ Agitada } \\
\hline Sim & $30(56,60)$ & $23(43,30)$ & 53 & & & & \\
\hline Não & $18(45)$ & $22(55)$ & 40 & $1,59(0,70-3,64)$ & 0,269 & - & - \\
\hline \multicolumn{8}{|l|}{ Calma } \\
\hline Sim & $19(41,30)$ & $27(58,70)$ & 46 & & & & \\
\hline Não & $29(61,70)$ & $18(38,30)$ & 47 & $0,43(0,19-1,00)$ & 0,051 & $0,31(0,12-0,79)$ & $0,014^{*}$ \\
\hline \multicolumn{8}{|l|}{ Hiperativa } \\
\hline Sim & $21(50)$ & $21(50)$ & 42 & & & & \\
\hline Não & $27(52,94)$ & $24(47,06)$ & 51 & $0,89(0,39-2,01)$ & 0,778 & - & - \\
\hline \multicolumn{8}{|l|}{ Ansiosa } \\
\hline Sim & $31(45,59)$ & $37(54,41)$ & 68 & & & & \\
\hline Não & $17(68)$ & $8(32)$ & 25 & $0,39(0,15-1,03)$ & 0,059 & $0,27(0,09-0,79)$ & $0,016^{*}$ \\
\hline \multicolumn{8}{|l|}{ Agressiva } \\
\hline Sim & $5(62,50)$ & $3(37,50)$ & 8 & & & & \\
\hline Não & $43(50,59)$ & $42(49,41)$ & 85 & $1,63(0,37-7,25)$ & 0,522 & - & - \\
\hline Total & $48(51,61)$ & $45(48,39)$ & 93 & & & & \\
\hline
\end{tabular}




\section{DISCUSSÃO}

Pesquisas epidemiológicas apontam um intervalo de frequência amplo no que diz respeito à presença de BS em crianças. Pesquisadores pedem mais atenção para a fragilidade do diagnóstico do bruxismo do sono, principalmente em crianças ${ }^{4,8}$. Levando em consideração que essa percepção vem de relato de terceiros e está sujeita a diversos fatores de influência e vieses é importante criar estratégias que possam minimizar tais vieses e ajudar essas famílias no diagnóstico e controle do bruxismo do sono. Na presente pesquisa, nossa ferramenta de diagnóstico foi o nível provável, onde além do relato do cuidador sobre a presença de sons ouvido através do ranger dos dentes, um exame clínico das facetas de desgaste foi realizado. Apenas um examinador realizou as entrevistas e o exame clínico ${ }^{9}$. No entanto, o foco principal de observação foi o relato dos pais e a associação com os traços de personalidade.

A percepção dos cuidadores em relação as características de personalidade dos filhos (características psicológicas), não guarda relação direta com presença ou ausência de bruxismo do sono. Existe uma crença de que crianças ansiosas tem mais bruxismo do sono, resultado não constatado na amostra do presente estudo ${ }^{10,11}$ Podemos com isso supor que a personalidade da criança não tem relação com o bruxismo do sono, confirmando o caráter sinalizador do bruxismo do sono em relação a alterações sistêmicas ${ }^{12}$. Por outro lado, caso o bruxismo do sono possa apresentar relação com aspectos psicológicos, a perspectiva dos pais em relação a isso não é confiável, pois nesse trabalho temos uma fragilidade da perspectiva dos pais em relação aos aspectos psicológicos, uma vez que crianças ansiosas apresentaram menor prevalência de bruxismo do sono assim como as crianças calmas. Isso pode confirmar a exclusividade da associação do bruxismo do sono as questões sistêmicas como alteração respiratória, substâncias psicoativas e refluxo gastresofágico.

As limitações do estudo são inerentes ao desenho da pesquisa, que é transversal e não fornecer associação de causalidade. Além disso, foi investigada uma amostra de conveniência, que frequenta regularmente clínicas odontológicas e assim, os cuidadores podem ter percepções em relação às questões de saúde diferente daqueles que não tem o hábito de levar as crianças para tratamento odontológico.

\section{CONCLUSÂO:}

As evidências sugerem que tanto as crianças consideradas calmas, quanto as ansiosas apresentaram menos relato de bruxismo do sono, demonstrando não haver nessa amostra, associação entre padrão de personalidade e bruxismo do sono. 


\section{REFERÊNCIAS}

1- Lobbezoo F, Ahlberg J, Raphael KG, Wetselaar P, Glaros AG, Kato T, Santiago V, Winocur E, De Laat A, De Leeuw R, Koyano K, Lavigne GJ, Svensson P, Manfredini $D$. International consensus on the assessment of bruxism: report of a work inprogress. J Oral Rehabil. 2018 nov; 45(11):837-844.

2- Lobbezoo F, Ahlberg J, Glaros AG, Kato T, Koyano K, Lavigne GJ, de Leeuw R, Manfredini D, Svensson P, Winocur E. Bruxism defined and graded: an international consensus. J Oral Rehabil. 2013; 40(1):2-4.

3- Carra MC, Huynh N, Lavigne G. Sleep bruxism: a comprehensive overview for the dental clinician interested in sleep medicine. Dent Clin North Am. 2012 Apr; 56(2):387-413.

4- Manfredini D, Restrepo C, Diaz-Serrano K, Winocur E, Lobbezoo F. Prevalence of sleep bruxism in children: a systematic review of the literature. J Oral Rehabil. 2013; 40(8):631-42.

5- Huynh NT, Desplats E, Bellerive A. Sleep bruxism in children: sleep studies correlate poorly with parental reports. Sleep Med. 2016; 19:63-8.

6- Stuginski-Barbosa J, Porporatti AL, Costa YM, Svensson P, Conti PC. Agreement of the International Classification of Sleep Disorders Criteria with polysomnography for sleep bruxism diagnosis: A preliminary study. J Prosthet Dent. 2017 Jan; 117(1):61-66.
7- Accinelli RA, Llanos O, López LM, Matayoshi S, Oros YP, Kheirandish-Gozal L,Gozal D. Caregiver perception of sleep-disordered breathing-associated symptoms in children of rural Andean communities above 4000 masl with chronic exposure to biomass fuel. Sleep Med. 2015; 16(6):723-8.

8- Restrepo C, Manfredini D, Castrillon E, Svensson P, Santamaria A, Alvarez C, Manrique R, Lobbezo F. Diagnostic Accuracy of the use of parental- reported sleep bruxism in a polysomnographic study in children. Int $\mathrm{J}$ Pediatr Dent. 2017; 27(5):318-325.

9- Bortoletto CC, Salgueiro MDCC, Valio R, Fragoso YD, Motta PB, Motta LJ, Kobayashy FY, Fernandes KPS, Mesquita- Ferrari RA, Deana A, Bussadori SK. The relationship between bruxism, sleep quality, and headaches in schoolchildren. J Phys Ther Sci. 2017 Nov; 29(11):1889-1892.

10- Obayashi K. Salivary mental stress proteins. Clin Chim Acta. 2013; 21(425):196-201.

11- Restrepo C, M. Alvarez \& I. Valencia. Personality traits and temporomandibular disorders in a group of children with bruxing behaviour. J Oral Rehabil. 2008; 35:585-93.

12- Mengatto CM, Dalberto Cda S, Scheeren B, Barros SG. Association between sleep bruxism and gastroesophageal reflux disease. J Prosthet Dent. 2013; 110(5):349-55. 